Influencia de la fragmentación del hábitat en la abundancia

de Mazama temama a diferentes escalas en un bosque mesófilo de montaña

\title{
Influence of habitat fragmentation on bundance of Mazama temama at different scales in the cloud forest
}

\author{
Brenda Muñoz-Vazquez ${ }^{1}$ y Sonia Gallina-Tessaro ${ }^{1^{*}}$
}

\begin{abstract}
${ }^{1}$ Red de Biología y Conservación de Vertebrados, Instituto de Ecología A. C. Carretera Antigua a Coatepec 351, El Haya 91000. Xalapa, Veracruz, México. E-mail: bren2801@gmail.com (BM-V), sonia.gallina@inecol.mx (SGT)

*Corresponding author
\end{abstract}

Brocket deer inhabit two of the most threatened ecosystems in Mexico: the cloud forest and tropical evergreen forest, due to the lack of studies little is known about their current condition. However it is believed that it is threatened by various factors among them hunting, loss and fragmentation of habitat. In this paper we established a conceptual model a priori about the relationships between deer and their habitat where we included elements of microhabitat and landscape to determine how it affects the abundance and distribution of brocket deer in the mountains of the state of Hidalgo, Mexico. We selected 14 different forested sites, in each we established two transects $250 \mathrm{~m}$ long where the relative abundance of tracks was estimated. During April 2011 to March 2012 three sampling visit were made in each transect. In the transects we measured: forest structure, protective cover, low tree and shrub layer. For each site we considered the size and shape of the fragments and their matrix. To determine which variables influenced the abundance of tracks we used general linear regression models. 352 brocket deer tracks were recorded in $21 \mathrm{~km}$ of linear transects. The average relative abundance was 16.76 tracks $/ \mathrm{km}$. More tracks were found in sites with higher tree density 0.4 trees $/ \mathrm{m} 2,60 \%$ canopy cover, $70 \%$ protection cover for fawns and $50 \%$ for adults, higher linear density 0.5 ind / $25 \mathrm{~m}$ and shrub richness of more than four species. The abundance of deer had a positive linear relationship with the density of tree coverage to protect adults and brocket deer importance value of edible trees $(P<0.001)$. The estimated abundance of tracks was higher than the reported in other sites. Our data suggest that the sites $C 2, C 4$ and $C 7$ were better suited for deer use and possibly their movements to other sites attracted by some crops, but more specific studies would be necessary like radio-telemetry to test this assumption. This research indicated that brocket deer behaves like a forest specialist, as their abundance and welfare depends on food availability, habitat quality in terms of forest cover and the remoteness of settlements, which are generally the best preserved and most inaccessible fragments.

El venado temazate habita en dos de los ecosistemas más amenazados en México: los bosques mesófilos de montaña y selvas. Debido a la falta de estudios poco se sabe acerca de su estado actual de conservación. Sin embargo, se cree que está amenazada por diversos factores entre los que destacan la cacería y la pérdida y fragmentación de su hábitat. En este trabajo establecimos un modelo conceptual a priori sobre las relaciones venado-hábitat donde incluimos elementos del microhábitat y del paisaje para determinar cómo afecta la fragmentación del paisaje y sus factores secundarios en la abundancia y distribución del temazate en las montañas de Hidalgo México. Elegimos 14 sitios con diferente cobertura forestal, en cada uno establecimos dos transectos en franja de 250 metros de largo por un metro de ancho, donde se calculó la abundancia relativa con conteo de rastros. Se realizaron tres visitas a cada transecto en franja durante el periodo abril 2011-marzo 2012. Además, se midió: estructura forestal, cobertura de protección, estrato arbustivo y arbóreo bajo. También para cada sitio analizamos la configuración de los fragmentos con mediciones del tamaño y forma de los fragmentos de bosque y su matriz. Realizamos modelos lineales de regresión múltiple para determinar que variables influían en la abundancia de rastros. Se registraron 352 rastros de venado temazate en $21 \mathrm{~km}$ lineales de transectos en franja. El índice de abundancia relativa promedio fue de $16.76 \mathrm{rastros} / \mathrm{km}$. Se observó mayor cantidad de rastros en sitios con densidad absoluta mayor a 0.4 árboles $/ \mathrm{m}^{2}$, cobertura de dosel mayor al $60 \%$, cobertura de protección a crías mayor al $70 \%$ y mayor a $50 \%$ para los adultos, una densidad lineal mayor a $0.5 \mathrm{ind} / 25 \mathrm{~m}$ y una riqueza de arbustivas mayor a cuatro especies. La abundancia de rastros de venado temazate tiene una relación lineal positiva con la densidad absoluta de árboles, cobertura de protección a venados temazate adultos y valor de importancia de árboles comestibles $(P<0.001)$. La abundancia promedio de rastros estimada para este estudio es mayor a la de otros sitios. Nuestros datos apuntan a que en conjunto los sitios C2, C4 y C7 albergan la mayor cantidad de individuos y que posiblemente se estén desplazando a los otros sitios atraídos por algunos cultivos, sin embargo serían necesarios estudios más específicos como los de radio telemetría para comprobar este supuesto. Los resultados de la investigación 
indican que el temazate se comporta como un especialista de interior de bosque, ya que su abundancia depende de la disponibilidad de alimento, la calidad del hábitat en cuanto a cobertura forestal y la lejanía de asentamientos humanos, que en general son los fragmentos mejor conservados y de más difícil acceso.

Key words: cloud forest; habitat fragmentation; hunting; Mazama temama; trails.

(C) 2016 Asociación Mexicana de Mastozoología, www.mastozoologiamexicana.org

\section{Introducción}

El venado temazate (Mazama temama, Geist 1998) es el cérvido más pequeño de México, el cual habita en los bosques mesófilos de montaña (BMM) y selvas desde el centro de dicho país y hasta el norte de Colombia. Estos son los dos ecosistemas más afectados por la larga historia de dominancia de la ganadería (aproximadamente el $50 \%$ de la superficie original de BMM ha desaparecido, Jones et al. 1983). Debido a la falta de estudios poco se sabe acerca del estado actual de conservación de las poblaciones de temazate, pero se piensa que están amenazadas por tratarse de una especie preferida por los cazadores, por ser vulnerable a la depredación por animales domésticos, particularmente perros ferales, y por la disminución y pérdida de su hábitat (Weber y González 2003; Mandujano 2011; Weber 2014).

Para ampliar las probabilidades de sobrevivencia de una especie dentro de un sitio es necesario tomar en cuenta dentro del plan de conservación el hábitat a diferentes escalas, especialmente en ambientes con algún grado de perturbación (Bennett et al.2006). En primer instancia es necesario considerar los elementos del microhábitat que influyen en el riesgo de depredación y la dinámica poblacional local (Bertolino 2007) y posteriormente la estructura del paisaje que limita el éxito de dispersión de las especies de manera más significativa en ambientes fragmentados (Brady et al. 2011). El presente estudio representa uno de los primeros esfuerzos por conocer el impacto que tienen los elementos del hábitat a distintas escalas espaciales en la abundancia y distribución del venado temazate rojo, pues los pocos estudios realizados hasta el momento solo evalúan alguno de estos aspectos.

El objetivo general fue determinar qué elementos del paisaje influyen en la abundancia y distribución del venado temazate. Para resolverlo se estableció un modelo conceptual a priori sobre las relaciones venado-hábitat que incluyó elementos del microhábitat y paisaje.

El modelo de microhábtat incluye características importantes para la incidencia y abundancia de venado temazate relacionadas directamente con la estructura vegetal, que influyen en la cobertura de protección y disponibilidad de alimento a nivel de sitio de estudio. Estos elementos contribuyen al bienestar de los venados al proveer protección frente a factores ambientales (sol, precipitación, temperatura, entre otros), los cuales incrementan las probabilidades de crear hábitat específicos que permiten al venado esconderse y escapar de sus depredadores, brindándoles la seguridad necesaria para mantener una buena condición física (Dasmann 1971). Particularmente el venado temazate, se comporta como especialista de hábitat en algunos lugares, además debido a que se trata de un rumiante pequeño y selectivo, requiere de mayor energía por unidad de peso corporal para satisfacer sus necesidades básicas en comparación con otros venados de mayor talla como el venado cola blanca y el venado bura (Paredes 2002; Bello et al. 2004; Villarreal-Espino et al. 2008).

El modelo de paisaje incluye elementos de fragmentación del hábitat y características de la matriz, en particular la presencia de asentamientos humanos y superficies desprovistas de vegetación primaria. Estos factores limitan la dispersión de algunas especies a través del paisaje, afectando la recolonización, favoreciendo su cacería y depredación por animales domésticos. 
En especial el venado temazate se caza por su alto valor nutrimental o por ser considerada una especie problema que afecta ciertos cultivos (Escamilla et al. 2000; Lopes y Ferrari 2000; Peres 2001; Reyna-Hurtado 2002; Reyna-Hurtado y Tanner 2007; Bello et al. 2010), y además se conoce que es un especialista de hábitat primario y la fragmentación del hábitat no lo favorece y limita su dispersión (Weber 2005; Reyna-Hurtado y Tanner 2005).

\section{Material y métodos}

Área de estudio. El estudio se llevó a cabo en el bosque mesófilo de montaña $(6,070.1 \mathrm{ha})$ del municipio de San Bartolo Tutotepec, Hidalgo, 20 24'50" N y -98 14' $24^{\prime \prime}$ O México (Figura 1). Esta zona pertenece a la reserva de biósfera corredor biológico del bosque mesófilo de montaña y se caracteriza por ser uno de los BMM mejor conservados en el estado. Posee elevaciones que van de los 200 a 1,944 m, presenta una topografía accidentada, con pendientes pronunciadas y escarpadas y predominan los suelos de tipo regosol: calcárico, dístrico y eútrico (CONABIO 2010). Se presentan tres zonas climáticas, cálidas, semicálidas y templadas, con un rango de temperatura media anual de 12 a $18{ }^{\circ} \mathrm{C}$ y un rango de precipitación anual de 1,200 a 2,000 mm (Villavicencio y Pérez-Escandón 2007). Las especies vegetales dominantes en la zona de estudio son Pinus teocote, Fagus grandifolia subsp. mexicana, Quercus xalapensis, Q. sartorii, Liquidambar styraciflua, Clethra macrophylla, Oreopanax xalapensis, Cyathea fulva y Dicksonia sellowiana en los fragmentos de bosque y en las zonas abiertas predominan Alnus acumminata y A. jorullensis.

Para seleccionar los sitios de muestreo utilizamos una imagen satelital pancromática tomada con el sensor Spot 5 en el año 2011, con una resolución espacial de 2.5 m (Scene ID: 5 590-309; (SPOTIMAGE et al. 2011). Con el programa arcview 3.2 trazamos 14 circunferencias de un kilómetro de diámetro que correspondían a nuestros 14 sitios de estudio, cada uno de ellos con diferente porcentaje de cobertura forestal (30 - $100 \%$ del círculo ocupado por bosque). Las circunferencias con una cobertura vegetal mayor al $60 \%$ fueron consideradas a priori como sitios conservados (C) y en caso contrario fueron consideradas como sitios perturbados (P).

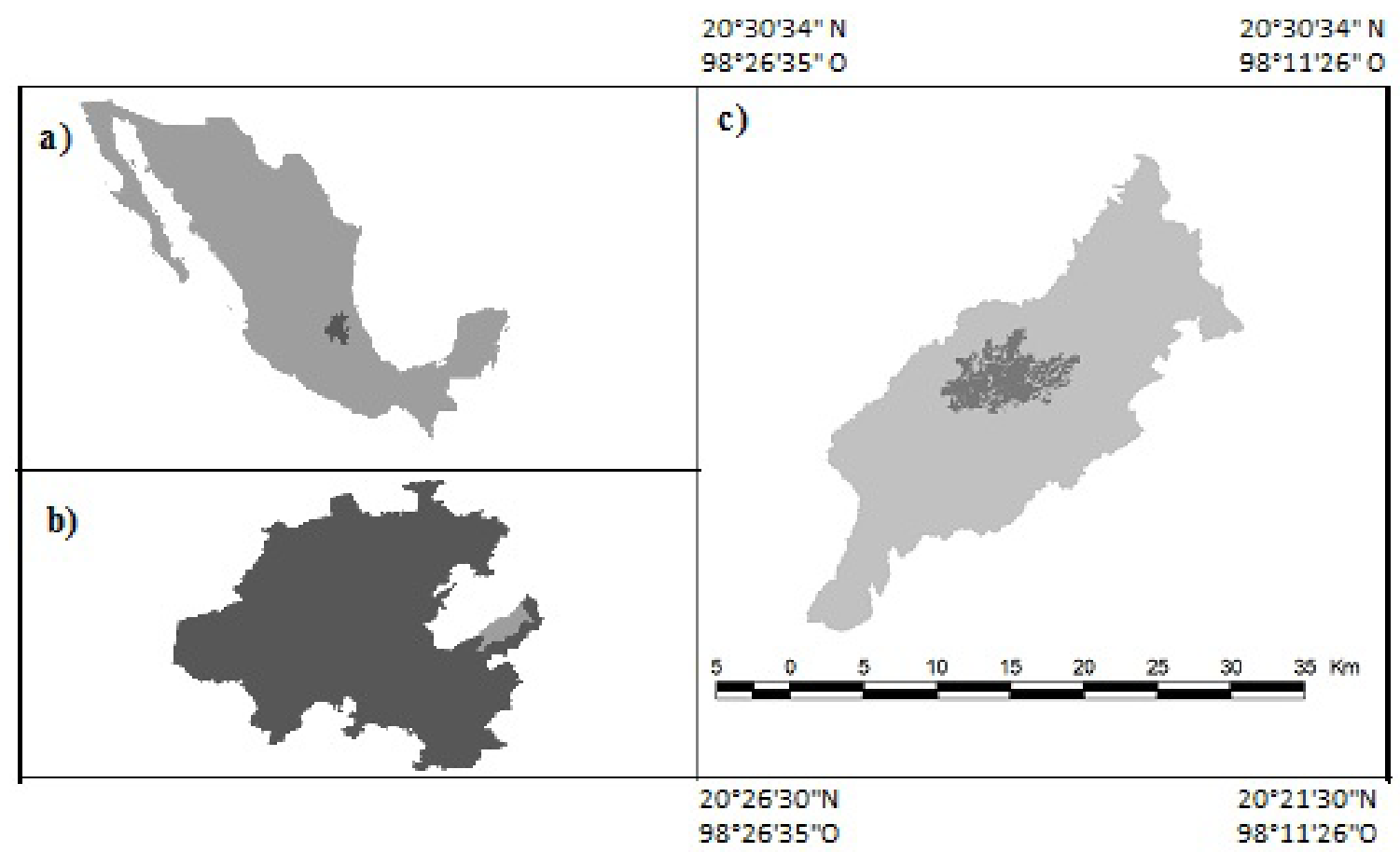

Figura 1. Ubicación geográfica de la zona de estudio en a) México, en el b) Estado de Hidalgo, c) en el municipio de San Bartolo Tutotepec (las partes sombreadas representan el área de estudio). 
Para estimar la abundancia relativa de rastros de venado temazate utilizamos el método de transecto en franja, con medidas de $250 \mathrm{~m}$ de largo por un metro de ancho dentro de cada sitio de muestreo, cada uno de ellos fue georreferenciado. Se establecieron dos transectos en franja en cada sitio de muestreo y estos se separaron de los transectos en franja de los otros sitios de muestreo por una distancia mínima de $1 \mathrm{~km}$ (Figura 2). Cada transecto en franja fue recorrido en tres ocasiones durante el periodo abril 2011-marzo 2012 a diferentes horas del día, entre las 8:00 y las 17:00 horas. Los registros como huellas, excretas, echaderos y comederos identificados fueron considerados como evidencia de la presencia del venado temazate a partir de la descripción de Aranda (2000), es importante mencionar que es la única especie de cérvido presente en la zona de estudio, por lo que no existe el riesgo de confundir sus rastros con los de otro como el venado cola blanca. Cada registro fue geo-referenciado con un GPS Garmin 60CSx e incorporado a un sistema de información geográfica utilizando el programa Arc view ver. 3.2. (ESRI 1999).

Se calculó el índice de abundancia relativa (IAR) por kilómetro con la siguiente fórmula: IAR = número de rastros/ unidad de muestreo $(0.5 \mathrm{~km})$. En los meses de abril y junio del $2011 \mathrm{se}$ establecieron 11 puntos dentro de cada transecto en franja establecido previamente para identificar la presencia de la especie, separados cada $50 \mathrm{~m}$ para evaluar 11 elementos del microhábitat: densidad absoluta de árboles (DAA), altura media de los árboles (AMA), área basal media de los árboles ( $A B A$ ), riqueza de especies forestales (REF) siguiendo el método de cuadrantes centrados en puntos propuesto por Müeller-Dombois y Ellenberg (1974), valor de importancia de las especies vegetales comestibles $(\mathrm{VIC})$ generado a partir de la comparación de la lista de especies vegetales obtenida en campo y la de Villarreal-Espino et al. 2008, la cobertura del dosel (CDD) con el método de fotografía digital de Kuusipalo (1985), Teraoka (1996) e Ishida (2004), la cobertura de protección para las crías de venado temazate (CPC) y la cobertura de protección para los venados temazates adultos (CPA) se obtuvo utilizando el método de cobertura vertical de Griffith y Youtie (1988) y por último las variables de riqueza de especies (REH), densidad lineal (DLH) y cobertura lineal (CLH) del

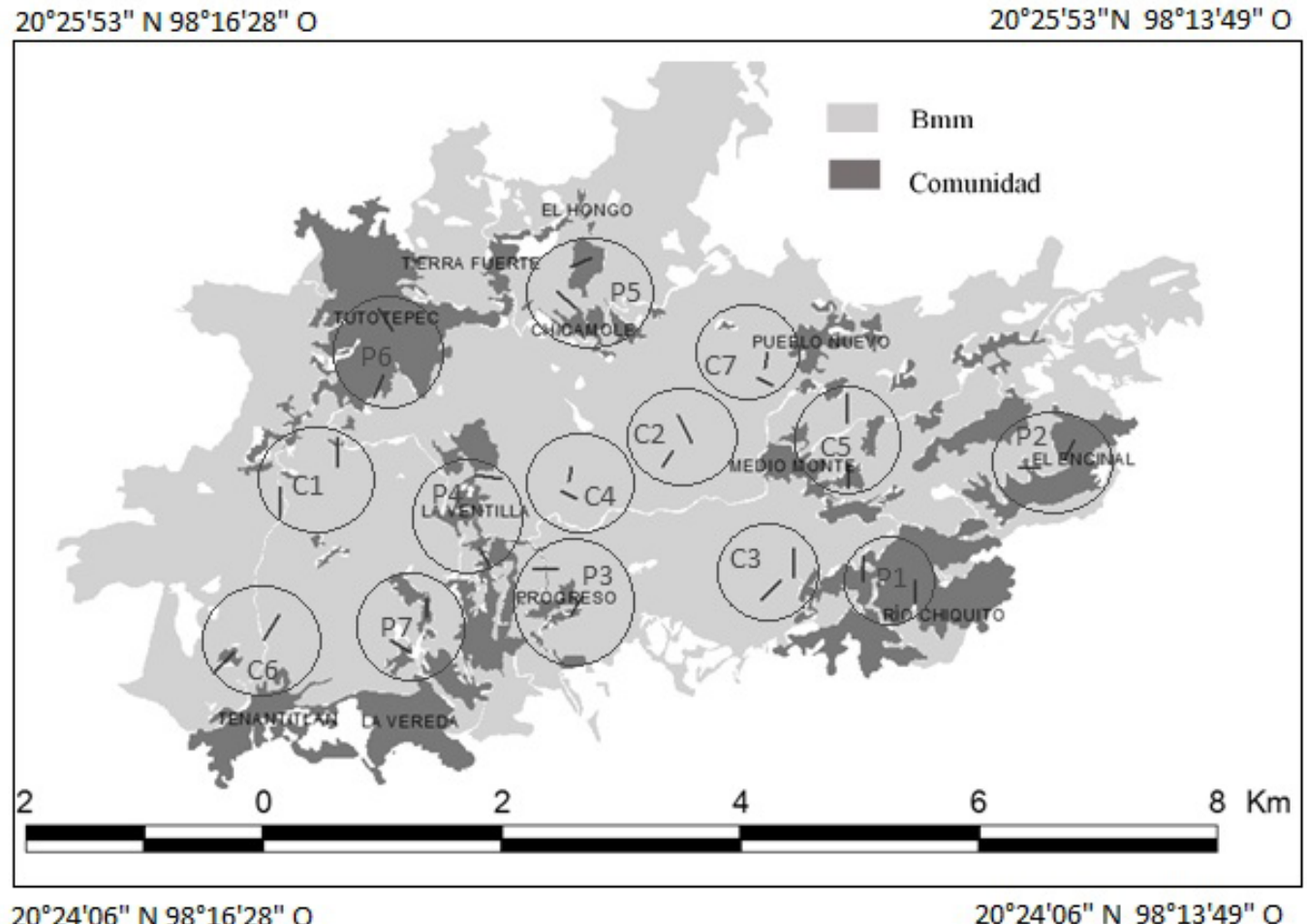

Figura 2. ubicación de los transectos en franja y los sitios de muestreo establecidos para rastrear al venado temazate con relación a las comunidades humanas de la zona. $B \mathrm{~mm}=$ bosque mesófilo de montaña; $\mathrm{C}=$ conservados; $\mathrm{P}=$ perturbados 
estrato arbustivo, herbáceo y arbóreo bajo se estimaron a partir del método de línea intercepción (Canfield 1941).

Para analizar la configuración de los fragmentos evaluamos para cada circunferencia siete elementos: área total de bosque (ATB), número de fragmentos (NFB), borde total (BTB), área núcleo total (ANT), índice de forma del paisaje (IFP), índice de proximidad (IPP) e índice de división (IDP; McGarigal et al. 2002), con el programa Frag Stats (USDA 1995).

Se eligieron nueve elementos para caracterizar la matriz en la que se encuentran embebidos los fragmentos de bosque, para esto utilizamos la imagen Spot previamente citada y con ayuda del programa Arc view ver. 3.2 (ESRI 1999), se crearon buffers de $500 \mathrm{~m}$ (B5M), 1 km (B1K), 2 km (B2K) y 4 km (B4K) alrededor de cada uno de los 14 transectos en franja ubicados dentro de las circunferencias. Sobre esta imagen se identificó la comunidad humana más cercana al transecto en franja y se calculó su área (ACC), el número de viviendas que la conformaban (NVC) y la distancia que había entre el transecto en franja y la vivienda más cercana (DMV), también se obtuvo su número de habitantes (NHC) y número de perros (NPC), estas dos últimas a partir del censo del año 2012 que realizan las casas de salud comunitarias. Las viviendas se tomaron como indicio de la presencia de cazadores potenciales debido a que se sabe que en la zona la gente no se desplaza mucho para cazar, se concentra solo en los alrededores (Muñoz 2013).

Análisis estadístico. Debido a que las variables tenían diferentes escalas de medición utilizamos una estandarización (transformación de los datos), esto con ayuda del programa Sigma Stat, posteriormente se generó una matriz de correlación para determinar cuáles y en qué grado las variables eran colineales. Cuando una correlación excedió a 0.8 la variable con menor significado biológico fue omitida del modelo. Para determinar cuáles eran las variables (independientes) que tenían influencia en la abundancia de rastros de venado temazate (variable de respuesta), se realizaron modelos lineales de regresión múltiple (GLMR) con análisis gradual posterior (forward stepwise), partiendo del modelo sin covariables, para cada uno de los niveles: microhábitat, fragmentos y matriz y un modelo general que incluía las variables de los tres niveles de paisaje. Se utilizó el programa Statistica (Stat Soft 2004), para llevar a cabo los análisis estadísticos.

\section{Resultados}

Se identificaron 352 registros de venado temazate con un esfuerzo de muestreo de $21 \mathrm{~km}$ lineales. El índice de abundancia relativa promedio fue de $16.76 \mathrm{rastros} / \mathrm{km}$. Se encontraron registros en casi todos los transectos en franja, con excepción del P7, detectando tres zonas de mayor concentración en los sitios C2, C4 y C7 (Tabla 1). La mayoría de los registros se obtuvieron dentro de zonas forestales alejadas de manchas urbanas, incluso en los sitios con mayor porcentaje de deforestación.

Se identificaron distintas asociaciones vegetales dominadas por: Pinus teocote (C1), Fagus grandifolia subsp. mexicana (C2), Quercus xalapensis (C3, C4, C5, C7), Liquidambar styraciflua (C6), Clethra macrophylla (P1), Buddleia cordarta (P2), Phaseolus vulgaris (P3, P5), Alnus jorullensis (P4, P6), Alnus acuminata (P7). En total se registraron 41 especies arbóreas, las cuales en su mayoría representaron los sitios conservados y 66 especies arbustivas. Se sabe que 12 de estas especies son consumidas por el temazate en la sierra de Puebla (Villarreal-Espino et al. 2008). 
Se observó una mayor cantidad de rastros, de manera estadísticamente significativa $(P<0.001)$, en los sitios con densidad absoluta mayor a 0.4 árboles $/ \mathrm{m}^{2}$, una cobertura de dosel mayor al $60 \%$, cobertura de protección a crías de venado temazate mayor al $70 \%$ y mayor a $50 \%$ en el caso de los temazates adultos, una densidad lineal mayor a $0.5 \mathrm{ind} / 25 \mathrm{~m}$ y una riqueza de arbustivas mayor a cuatro especies.

Debido a la alta colinealidad entre las variables se utilizaron solamente 14 de las 27 medidas (Tabla 2). Con el primer modelo determinamos que la abundancia de rastros puede explicarse en un $95 \%$ por la densidad absoluta de árboles, el valor de importancia de especies vegetales comestibles y la cobertura de protección para temazates adultos, el segundo modelo nos explica $70 \%$ de la variabilidad de la abundancia de rastros con el área núcleo total y el índice de proximidad, el modelo que incluye las variables de la matriz nos explica $70 \%$ de la variabilidad por medio de un solo elemento, la distancia mínima a las viviendas (Tabla 3).

A partir de un análisis general entre los tres niveles de variables ambientales: microhábitat, fragmentos y matriz se registró que la variabilidad en la abundancia de rastros de venado temazate puede explicarse con tres variables principales: densidad absoluta de árboles, el valor de importancia de especies vegetales comestibles y la cobertura de protección para temazates adultos.

\section{Discusión}

La abundancia promedio de rastros estimada para este estudio es alta en comparación con la reportada por otros trabajos en BMM (0.38 rastros/km; Lira y Naranjo 2003) y en bosque tropical $(0.29,0.12,2.36,0.1,5.1$ rastros $/ \mathrm{km}$; Bolaños y Naranjo 2001; Bello et al. 2004; Martínez-Kú et al. 2008; Naranjo 2008; Reyna y Tanner 2010). Esto probablemente se deba a que tiene pocos competidores, en esta zona no ocurre ninguna otra especie de venado y su depredador natural que en ese caso sería el puma se encuentra extinto localmente (B. Muñoz, obs. pers.).

Tabla 1. Número de rastros y abundancia relativa $($ rastros $/ \mathrm{km})$ de venado temazate en cada transecto en franja.

\begin{tabular}{lrrrrrrr}
\hline Transecto & Hu & Ex & Ec & Co & Total & \multicolumn{2}{c}{ lar } \\
\hline C1 & 27 & 2 & 1 & 0 & 30 & 20.0 \\
C2 & 46 & 0 & 0 & 1 & 47 & 31.3 \\
C3 & 45 & 0 & 0 & 0 & 45 & 30.0 \\
C4 & 112 & 1 & 0 & 1 & 114 & 76.0 \\
C5 & 17 & 0 & 0 & 0 & 17 & 11.3 \\
C6 & 31 & 0 & 0 & 0 & 31 & 20.7 \\
C7 & 43 & 0 & 0 & 0 & 43 & 28.7 \\
P1 & 4 & 0 & 0 & 0 & 4 & 2.7 \\
P2 & 7 & 0 & 0 & 0 & 7 & 4.7 \\
P3 & 2 & 0 & 0 & 0 & 2 & 1.3 \\
P4 & 2 & 0 & 0 & 0 & 2 & 1.3 \\
P5 & 9 & 0 & 0 & 0 & 9 & 6.0 \\
P6 & 1 & 0 & 0 & 0 & 1 & 0.7 \\
P7 & 0 & 0 & 0 & 0 & 0 & 0.0 \\
& & & & & & & \\
\end{tabular}

$\mathrm{C}=$ sitios conservados; $\mathrm{P}=$ sitios perturbados; $\mathrm{Hu}=$ huellas; $\mathrm{Ex}=$ excretas; $\mathrm{Ec}=$ echaderos; $\mathrm{Co}=$ comederos; lar = índice de abundancia relativa. 
Tabla 2. Variables tomadas en cuenta para el modelo general de regresión

\begin{tabular}{|c|c|c|c|c|c|c|c|c|c|c|c|c|c|c|}
\hline \multirow[b]{2}{*}{ SITIO } & \multicolumn{5}{|c|}{ MICROHÁBITAT } & \multicolumn{3}{|c|}{ FRAGMENTOS } & \multicolumn{6}{|c|}{ MATRIZ } \\
\hline & DAA & $\begin{array}{l}\underset{(x 103)}{V} \text { I C }\end{array}$ & CPA & $\mathrm{CLH}$ & REH & $\begin{array}{l}\text { B T B } \\
(x 103)\end{array}$ & ANT & $\underset{(x 103)}{P}$ & $\mathrm{NHC}$ & ACC & B5M & B1K & B4K & DMV \\
\hline $\mathrm{C} 1$ & 27.89 & 9.09 & 80.3 & 0.42 & 12 & 5.25 & 41.9 & N/A & 258 & 144 & 0 & 21 & 408 & 500.3 \\
\hline $\mathrm{C} 2$ & 61.93 & 1.34 & 71.7 & 0.23 & 16 & 3.96 & 50.4 & N/A & 161 & 13 & 0 & 5 & 467 & 830.2 \\
\hline C3 & 34.37 & 14.98 & 73.9 & 0.6 & 17 & 7.63 & 29.1 & N/A & 230 & 118 & 4 & 25 & 617 & 417.4 \\
\hline C4 & 81.79 & 61.08 & 56.5 & 0.61 & 18 & 5.42 & 44.6 & 6.29 & 40 & 29.4 & 0 & 3 & 413 & 884.4 \\
\hline C5 & 24.81 & 2.13 & 76.8 & 0.36 & 15 & 13.43 & 3.71 & 67.14 & 34.1 & 69 & 5 & 29 & 552 & 311.1 \\
\hline C6 & 32.76 & 0.12 & 79.4 & 0.91 & 22 & 8.09 & 13.9 & 44.24 & 46.4 & 214 & 3 & 10 & 443 & 417.4 \\
\hline C7 & 64.39 & 11.86 & 58.8 & 0.69 & 20 & 5.05 & 37.2 & N/A & 21.4 & 136 & 11 & 28 & 499 & 362.8 \\
\hline P1 & 4.95 & 0.28 & 36.1 & 0.58 & 8 & 14.50 & 0.01 & 1.77 & 230 & 118 & 25 & 59 & 1029 & 99.6 \\
\hline P2 & 3.96 & 0.03 & 40 & 1.07 & 5 & 13.81 & 1.77 & 8.93 & 86 & 77.5 & 6 & 24 & 994 & 248.9 \\
\hline P3 & 2.12 & 0.009 & 3.03 & 0.68 & 16 & 10.35 & 16.9 & 7.13 & 54 & 14.5 & 3 & 26 & 425 & 211.2 \\
\hline P4 & 27.89 & 0.04 & 20 & 1.19 & 8 & 11.86 & 10.9 & 231.31 & 40 & 29.4 & 2 & 11 & 340 & 401.3 \\
\hline P5 & 0.71 & 0.41 & 28.8 & 1.16 & 15 & 11.62 & 8.68 & 24.20 & 161 & 13 & 3 & 15 & 428 & 194.1 \\
\hline P6 & 2.18 & 3.93 & 24.2 & 0.4 & 9 & 11.27 & 0.76 & 0.51 & 258 & 144 & 24 & 61 & 333 & 204.2 \\
\hline P7 & 7.54 & 0.88 & 31.8 & 2.83 & 9 & 15.49 & 0.83 & 23.21 & 92 & 87 & 19 & 54 & 356 & 175.2 \\
\hline
\end{tabular}

$\mathrm{C}=$ conservado; $\mathrm{P}=$ perturbado; $\mathrm{DAA}=$ densidad absoluta de árboles $\mathrm{VIC}=$ valor de importancia de las especies vegetales comestibles; $\mathrm{CPA}=$ cobertura de protección para los venados temazates adultos; $\mathrm{CLH}=$ cobertura lineal del estrato arbustivo, herbáceo y arbóreo bajo; $\mathrm{REH}=$ riqueza de especies del estrato arbustivo, herbáceo y arbóreo bajo; $\mathrm{BTB}=$ borde total; ANT = área núcleo total; IPP = Índice de proximidad; NHC = número de habitantes de la comunidad; $\mathrm{ACC}=$ área de la comunidad más cercana; B5M = buffer de $500 \mathrm{~m}$; B1K = buffer de $1 \mathrm{~km}$; B4K =buffer de $4 \mathrm{~km}$; DMV = distancia entre el transecto y la vivienda más cercana.

Nuestros datos apuntan a que en conjunto los sitios C2, C4 y C7 albergan la mayor cantidad de individuos y posteriormente se estén desplazando a los otros sitios atraídos por algunos cultivos de maíz, frijol y aguacate cercanas a zonas conservadas, como se ha registrado en otras zonas de distribución del temazate (Méndez y Bello 2005). Los sitios con mayor cobertura forestal le proporcionan al temazate un hábitat de buena calidad, debido a la presencia de un alto valor de importancia de las especies que se han comprobado como alimento para el temazate, altos porcentajes de cobertura de protección tanto para crías como para adultos y la mayor cobertura de dosel comparado con los otros sitios.

Estudios previos sobre el efecto de la fragmentación del hábitat y la cacería en la abundancia de venado temazate indican que son relativamente tolerantes a estos cambios (Urquiza-Haas et al. 2010; Thorton et al 2011) pero otros han detectado un efecto negativo y consideran al temazate como un especialista de bosque porque difícilmente se encuentra en vegetación secundaria y tiene una dieta muy especializada, constituida en su mayoría por frutos (Escamilla et al. 2000; ReynaHurtado y Tanner 2005; Weber 2005, 2008; Weber 2014)

Asimismo, los resultados de esta investigación indican que el temazate se comporta como un especialista de interior de bosque, ya que su abundancia depende de la disponibilidad de alimento, la calidad del hábitat en cuanto a cobertura forestal y la lejanía de asentamientos humanos, que en general son los fragmentos mejor conservados y de más difícil acceso. Algunos autores describen tres posibles causas para este comportamiento: los mamíferos con amplias áreas de actividad tienen dificultad para usar ambientes con muchos fragmentos pequeños y prefieren los 
continuos de bosque, los paisajes con fragmentos pequeños son hábitats de baja calidad y tienen mayor efecto de borde, la presencia humana es intensa en los bordes y disminuye conforme se avanza al interior del bosque, ya que el acceso es difícil (Thorton et al 2011).

Es difícil separar completamente los efectos específicos de la fragmentación del hábitat, la cacería y la estructura de la vegetación debido a que se encuentran correlacionados entre sí (Lopes y Ferrari 2000). Sin embargo, se conoce que la susceptibilidad de las especies a la cacería difiere entre bosques continuos y fragmentados, de hecho, la resilencia de algunas especies puede alterarse cuando los fragmentos son pequeños, esto debido a que generalmente los límites del bosque determinan el área de actividad de los individuos y en sitios donde la tasa de inmigración es baja y la presión de cacería es intensa existen más probabilidades de extinción local (Pulliam 1988; Cullen 2000).

Por lo tanto, algunos parches en los bosques mesófilos de montaña de San Bartolo Tutotepec representan un refugio para las poblaciones de venado temazate menos estudiadas y de distribución más norteña en el país por lo que tomar acciones de conservación resultaría en beneficio de la especie. La presencia humana en estos bosques es inevitable, el hombre ha actuado como depredador, competidory dispersor de semillas desde hace por lo menos 12,000 años en los bosques neotropicales (Balee 1994) sin embargo consideramos como medidas de conservación el control de la cacería del temazate en la zona e implementar programas de educación ambiental que ayuden a concientizar a la población a cerca de la importancia de conservar esta especie y los beneficios que esto conllevaría y probablemente así se podría evitar su extinción local a mediano plazo.

\section{Agradecimientos}

A la Comisión Nacional de Ciencia y Tecnología por la beca otorgada para estudios de maestría, al Instituto de Ecología, a G. Sánchez Rojas por su apoyo en las salidas de campo y sus comentarios que enriquecieron el trabajo, a J. Laborde Dovalí por sus valiosas aportaciones, a los técnicos de la red de Biología y Conservación de Vertebrados R. González Trápaga y A. Vázquez, a E. C. Rodríguez Ramírez por su importante colaboración en el proyecto, a R. Landgrave por proporcionarnos las imágenes satelitales SPOT y por último a los habitantes de las comunidades estudiadas, en particular a E. Lucio Garcia y su familia.

Tabla 3. Resultados del modelo lineal de regresión

\begin{tabular}{lllll}
\hline Nivel de paisaje & Efecto & g.l. & $\boldsymbol{F}$ & $\boldsymbol{p}$ \\
\hline \multirow{4}{*}{ Microambiental } & VIC & 1 & 50.08143 & 0.000034 \\
& DAA & 1 & 13.55838 & 0.004231 \\
Fragmentos & CPA & 1 & 5.83287 & 0.036363 \\
Matriz & ANT & 1 & 29.87196 & 0.000196 \\
& IPP & 1 & 6.98485 & 0.022875 \\
& DMV & 1 & 32.15291 & 0.000104 \\
Todos los niveles & VIC & 1 & 50.08143 & 0.000034 \\
& DAA & 1 & 13.55838 & 0.004231 \\
& CPA & 1 & 5.83287 & 0.036363 \\
\hline
\end{tabular}




\section{Literatura citada}

Aranda, M. 2000. Huellas y otros rastros de los mamíferos de talla grande y mediana de México. Conabio. Instituto de Ecología A. C. Xalapa, México.

BaLeE, W. 1994. Footprints of the forest: Kaapor ethnobotany-the historical ecology of plant utilization by an Amazonian people. Columbia University Press. New York, EE. UU.

Bello, J., Guzmán-Aguirre, C., y C. Chablé-Montero. 2004. Caracterización del habitat de tres especies de artiodáctilos en un área fragmentada de Tabasco México. Memorias VI congreso internacional sobre manejo de fauna silvestre en la Amazonía y América latina. lquitos, Perú.

Bello, J., R. Reyna-Hurtado, y J. Wilham. 2010. Central American Red Brocket Deer Mazama temama Kerr 1792. Pp. 166-171 en Neotropical cervidology: biology and medicine of Latin American deer. (Duarte, J. M. B y S. González eds.). IUCN/FUNEP.

Bennett, A. F., J. Q. Radford, y A. Haslem. 2006. Properties of land mosaics: implications for nature conservation in agricultural environments. Biological Conservation 133:250-264.

Bertolino, S. J. 2007. Microhabitat use by garden dormice during nocturnal activity. Journal of Zoology 272:176-182.

Bolaños, J. E., y E. Naranjo. 2001. Abundancia, densidad y distribución de las poblaciones de ungulados en la cuenca del Río Lacantún, Chiapas, México. Revista mexicana de Mastozoología 5:45-57.

Brady, M. J., C. A. Mcalpine, H. P. Possingham, C. J. Miller, y G. S. Baxter. 2011. Matrix is important for mammals in landscapes with small amounts of native forest habitat. Landscape Ecology 26:617-628.

Canfield, R. 1941. Application of the Line Interception Method in Sampling Range Vegetation. Journal of Forestry 39:388-394.

Conabio. 2010. El Bosque Mesófilo de Montaña en México: Amenazas y Oportunidades para su Conservación y Manejo Sostenible. Ciudad de México, México.

Cullen, L. R., R. E. Bodmer, y C. Valladares-P'adua. 2000. Effects of hunting in habitat fragments of the Atlantic Forests, Brazil. Biological Conservation 95:49-56.

Daniel, W. S., y D. B. Frels. 1971. A track-count method for censusing white-tailed deer. Austin Texas Parks and Wildlife Department. Austin Texas, EE.UU

Dasmann, W. 1971. If deer are to survive. Harrisburg: Stackpole books. Pennsylvania. EE.UU.

Escamilla, A., M. Sanvicente, M. Sosa , Y C. Galindo-Leal. 2000. Habitat Mosaic, Wildlife Availability, and Hunting in the Tropical Forest of Calakmul, Mexico. Conservation Biology 146:1592-1601.

ESRI (Environmental System Research Institute). 1999. ArcView GIS v 3.2. New York, EE.UU.

GeISt, V. P. 1998. Deer of the World, Their Evolution, Behavior y Ecology. Stackpole Books, Pennsylvania. EE.UU.

Griffith, B., y B.A. Youtie. 1988. Two devices for estimating foliage density and deer hiding cover. Wildlife society bulletin 16:206-210.

IsHIDA, M. 2004. Automatic thresholding for digital hemispherical photography. Canadian Journal of Forest Research, 34: 2208-2216.

Jones, J. K., D. C. Carter, y W. D. Webster. 1983. Records of Mammals from Hidalgo, Mexico. The Southwestern Naturalist 283:378-380.

Kuusipalo, J. 1985. On the use of tree stand parameters in estimating light conditions below the canopy. Silva Fennica 192:185-196.

Lira, I., y E. Naranjo. 2003. Abundancia, preferencias de hábitat e impacto del ecoturismo sobre el puma y dos de sus presas en la reserva de la biósfera El Triunfo, Chiapas, México. Revista mexicana de Mastozoología 7:20-39. 
Lopes, M. A., y S. F. Ferrari. 2000. Effects of Human Colonization on the Abundance and Diversity of Mammals in Eastern Brasilian Amazonia. Conservation Biology 146:16581665.

Mandujano, S. 2011. Bibliografía estudios de venados en México. Vol. 2: Instituto literario de Veracruz S. C. Xalapa, México.

Martínez-Kú, D. H., G. Escalona-Segura y J. A. Vargas-Contreras. 2008. Importancia de las aguadas para los mamíferos de talla mediana y grande en Calakmul, Campeche, México. Pp. 449-468 en Avances en el Estudio de los Mamíferos de México Vol. 2 (Lorenzo, C., E. Espinoza y J. Ortega, eds.). Asociación Mexicana de Mastozoología, A. C. Distrito Federal, México.

McGarigal, K., S. A. Cushman, M. C. Neel, y E. Ene. 2002. FRAGSTATS: Spatial Pattern Analysis Program for Categorical Maps. Amherst: University of Massachusetts. Recuperado de www.umass.edu/landeco/research/fragstats/fragstats

Méndez, S. M., y J. Bello. 2005. Daños a los cultivos de frijol, por mamíferos silvestres, en el ejido Agua Blanca, Tacotalpa, Tabasco, México. Trabajo presentado en la semana de divulgación y video científico. Universidad Juárez Autónoma de Tabasco, Villahermosa, México.

Müeller-Dombois, D., y H. Ellenberg. 1974. Aims and methods of vegetation ecology. John Wiley and Sons. New York, EE. UU.

MuÑoz, B. 2013. Distribución, abundancia y uso de hábitat del venado temazate (Mazama temama) en los bosques mesófilos de San Bartolo, Tutotepec, Hidalgo, México. Tesis de maestría. Instituto de Ecología, A.C., Xalapa, México.

Naranjo, E. 2008. Uso y conservación de mamíferos en la selva Lacandona, Chiapas, México. Pp. 675-691 en Avances en el Estudio de los Mamíferos de México Vol. 2. (Lorenzo, C., E. Espinoza, y J. Ortega, eds.). Asociación Mexicana de Mastozoología, A. C. Distrito Federal, México.

Neff, D. J. 1968. The Pellet-Group Count Technique for Big Game Trend, Census, and Distribution: A Review. The Journal of Wildlife Management 323:597-614.

Paredes, G. J. 2002. Manejo integral del venado Temazate Mazama americana en el zoólogico de Chapultepec. Trabajo presentado en el VIII Simposio sobre Venados en México "Ing. Jorge Villarreal González".

Peres, C. A. 2001. Synergistic effects of subsistence hunting and hábitat fragmentation on Amazonian forest vertebrate. Conservation Biology 156:1490-1505.

Puluiam, H. R. 1988. Sources, sinks, and population regulation. American Naturalist 132:652661

Reyna-Hurtado, R. 2002. Hunting effects on the ungulate species in Calakmul, Forest, Mexico. Tesis de Maestría. University of Florida. Florida, EE.UU.

Reyna-Hurtado, R., Y G. W. Tanner. 2007. Ungulate relative abundance in hunted and nonhunted sites in Calakmul Forest Southern Mexico. Biodiversity and Conservation 163: 743-756.

Reyna-Hurtado, R., Y G. W. Tanner. 2010. Efecto de la perturbación humana en la abundancia relativa de ungulados en tres comunidades de la región de Calakmul, Campeche, México. Pp. 115-135 en Uso y manejo de fauna Silvestre en el norte de Mesoamérica (Guerra, M. M., S. Calmé, S. Gallina, y E. Naranjo, eds.). Secretaría de Educación de Veracruz. Xalapa, México.

SpotimAGe, SAGARPA, SIAP, ERMEXS, INEGI, Y SEMAR. 2011. Imagen de satélite Spot J-K: Proporcionada por la secretaría de marina.

Stat Soft, I. 2004. STATISTICA data analysis software system Version 7. Recuperado de 
Www.statsoft.com

Teraoka, Y. 1996. An effective mensuration technique for multipurpose management using fish-eye photographic images. En Remote sensing and computer technology for natural resource assessment. (Saramaki, J., B. Koch, y H. G. Lund, eds.). University of Joensuu, Faculty of Forestry.

Thorton, D., L. C. Branch, y M. E. Sunquist. 2011. The relative influence of habitat loss and fragmentation: Do tropical mammals meet the temperate paradigm? Ecological Applications 216:2324-2333.

Urquiza-HaAs, T., C. A. Peres, y P. M. Dolman. 2010. Large vertebrate responses to forest cover and hunting pressure in communal landholdings and protected areas of the Yucatan Peninsula, Mexico. Animal Conservation 14:271-282.

Usda, Pacific Northwest Research Station. 1995. FRAGSTATS: Spatial pattern analysis program for quantifying landscape structure, technical report PNW-GTR-351.

Villarreal-Espino, O. A., L. E. Campos-Armendia, T. A. Castillo-Martínez, I. Cortes-Mena, F. X. Plata-Pérez, y G. D. Mendoza-Martínez. 2008. Composición botánica de la dieta del venado temazate rojo Mazama temama, en la sierra nororiental del estado de Puebla. Universidad y Ciencia 24:183-188.

Villavicencio, M. S., y B. E. Pérez-Escandón. 2007. Flora útil de la Huasteca y la zona otomítepehua de Hidalgo. En estudio biológico de las áreas naturales y reserva de la biósfera del estado de Hidalgo (López-Escamilla, A. L. y G. Pulido-Flores, eds.). Universidad Autónoma del Estado de Hidalgo. Pachuca, México.

Weber, M. 2005. Ecology and conservation of sympatric tropical deer populations in the Greater Calakmul Region, south-eastern Mexico. Doctor of Philosophy, University of Durham. Durham, United Kingdom.

WEBER, M. 2008. Un especialista, un generalista y un oportunista: uso de tipos de vegetación por tres especies de venados en Calakmul, Campeche. Pp. 483-496. En Avances en el Estudio de los Mamíferos de México Vol. 2. (Lorenzo, C., E. Espinoza, y J. Ortega, eds.). Asociación Mexicana de Mastozoología, A. C. Distrito Federal, México.

Weber, M., y S. González. 2003. Latin american deer diversity and conservation: a review of status and distribution. Écoscience 104:443-454.

Weber, M. 2014. Temazates y venado cola blanca tropicales. En: Ecología y manejo de fauna silvestre en México (Valdez, R. y J. A Ortega-S, eds.). Colegio de postgraduados. Texcoco, México.

Submited: October 13, 2015

Reviewed: December 3, 2016

Accepted: January 26, 2016

Associated editor: Rafael Reyna 
88 THERYA Vol.7 (1):77-87 